\title{
Vertical Visual Features Have a Strong Influence on Cuttlefish Camouflage
}

\author{
K. M. ULMER ${ }^{1, *}$, K. C. BURESCH${ }^{1}$, M. M. KOSSODO ${ }^{1}$, L. M. MÄTHGER ${ }^{1}$, \\ L. A. SIEMANN $^{1}$, AND R. T. HANLON ${ }^{1,2}$ \\ ${ }^{1}$ Program in Sensory Physiology and Behavior, Marine Biological Laboratory, Woods Hole, \\ Massachusetts 02543; and ${ }^{2}$ Ecology and Evolutionary Biology, Brown University, Providence, \\ Rhode Island 02906
}

\begin{abstract}
Cuttlefish and other cephalopods use visual cues from their surroundings to adaptively change their body pattern for camouflage. Numerous previous experiments have demonstrated the influence of two-dimensional (2D) substrates (e.g., sand and gravel habitats) on camouflage, yet many marine habitats have varied three-dimensional (3D) structures among which cuttlefish camouflage from predators, including benthic predators that view cuttlefish horizontally against such 3D backgrounds. We conducted laboratory experiments, using Sepia officinalis, to test the relative influence of horizontal versus vertical visual cues on cuttlefish camouflage: 2D patterns on benthic substrates were tested versus 2D wall patterns and 3D objects with patterns. Specifically, we investigated the influence of (i) quantity and (ii) placement of high-contrast elements on a 3D object or a 2D wall, as well as (iii) the diameter and (iv) number of $3 \mathrm{D}$ objects with high-contrast elements on cuttlefish body pattern expression. Additionally, we tested the influence of high-contrast visual stimuli covering the entire 2D benthic substrate versus the entire 2D wall. In all experiments, visual cues presented in the vertical plane evoked the strongest body pattern response in cuttlefish. These experiments support field observations that, in some marine habitats, cuttlefish will respond to vertically oriented background features even when the preponderance of visual information in their field of view seems to be from the 2D surrounding substrate. Such choices highlight the selective decision-making that occurs in cephalopods with their adaptive camouflage capability.
\end{abstract}

Received 24 January 2013; accepted 25 March 2013.

* To whom correspondence should be addressed. E-mail: kulmer@ mbl.edu

\section{Introduction}

Cuttlefish, such as Sepia officinalis Linnaeus, 1758, adaptively and dynamically change their body pattern for camouflage (Hanlon and Messenger, 1988, 1996; Shohet et al., 2006; Kelman et al., 2007; Mäthger et al., 2007; Allen et al., 2009; Zylinski et al., 2009a, b; Barbosa et al., 2012). Cuttlefish inhabit many different environments, including sandy open plains, rocky reef habitats, seagrass beds, and coral reefs, and are thus exposed to a variety of visual information (Boletzky, 1983; Hanlon and Messenger, 1996). These benthic animals dwell primarily on the seafloor, where they use information from the substrate (horizontal cues-e.g., sand, gravel) as well as from vertical facades (e.g., wall or rock face) and discrete 3D objects (e.g., rocks, algae clumps) in their immediate surroundings to adapt their appearance for camouflage (Barbosa et al., 2008a; Buresch et al., 2011). How cuttlefish respond to vertically oriented visual cues versus horizontal cues was investigated initially by Barbosa et al. (2008a), but here the relative influence of each is tested in detail.

Cuttlefish use three basic body pattern types for camouflage: Uniform, Mottle, and Disruptive (for a description of each body pattern type see Hanlon and Messenger, 1988) with variations on each pattern type (Hanlon et al., 2009). In the present experiments, we used large-scale, high-contrast stimuli (known to elicit the Disruptive body pattern) to investigate the body pattern response of S. officinalis. We chose to use stimuli that evoke a Disruptive body pattern because the visual cues used to evoke this pattern have been studied extensively (Chiao and Hanlon, 2001a, b; Mäthger et al., 2006, 2007; Barbosa et al., 2007, 2008a; Chiao et al., 2007, 2009; Kelman et al., 2007; Zylinski et al., 2009c).

Background matching and masquerade are two camou- 
flage tactics used by cuttlefish in the wild (Hanlon et al., 2009). Background matching functions by generally resembling the visual background seen by predators (Cott, 1940; Endler, 1978, 1981; Stevens and Merilaita, 2009). For masquerade, an animal resembles inanimate, inedible, or uninteresting objects in the immediate surroundings (such as twigs, leaves, stones, or seaweed) (Thayer and Thayer, 1918; Stevens and Merilaita, 2009; Skelhorn et al., 2010a), and many taxa use this camouflage tactic (e.g., stick insects, catepillars, lizards: Schmidt, 1990; Skelhorn et al., 2010b; Cooper and Sherbrooke, 2012). Whereas background matching hinders detection by a predator, masquerade prevents correct identification (Endler, 1981; Ruxton et al., 2005; Skelhorn et al., 2010a, b, 2011; Skelhorn and Ruxton, 2011; Stevens and Merilaita, 2011). In the wild, cuttlefish sometimes choose to masquerade as $3 \mathrm{D}$ objects such as rocks or clumps of algae by tuning their skin pattern, color, brightness, and arm posture to resemble the 3D object instead of the benthic substrate (Hanlon and Messenger, 1988; Barbosa et al., 2008a; Hanlon et al., 2009).

Recent laboratory experiments have suggested that cuttlefish will masquerade as a $3 \mathrm{D}$ object in the presence of a high-contrast 3D stimulus on a uniform 2D substrate $(\mathrm{Bu}-$ resch et al., 2011). Here, we examined this capability in greater detail by testing which characteristics of high-contrast 3D objects are most important in evoking a Disruptive body pattern, and whether body pattern expression evoked by the $3 \mathrm{D}$ stimuli could also be evoked using only 2D vertical stimuli with the same characteristics as the $3 \mathrm{D}$ freestanding objects.

\section{Materials and Methods}

\section{Animals}

European cuttlefish (Sepia officinalis) were hatched, reared, and maintained in the Marine Resources Center facility at the Marine Biological Laboratory in Woods Hole, Massachusetts. Ten animals (average mantle length (ML) $22 \mathrm{~mm}, \mathrm{SD}=3.35$; average White Square area $37 \mathrm{~mm}^{2}$, $\mathrm{SD}=7.63)$ were used for all of the experiments.

\section{Experimental setup}

Experiments were conducted inside a black tent to prevent disturbances during trials. Cuttlefish were observed on a TV monitor outside of the tent, and images were taken remotely. Animals were placed inside a circular arena (25-cm diameter) with flow-through seawater where they were presented with each stimulus, either 3D objects freestanding on the floor of the arena or $2 \mathrm{D}$ cues placed vertically on the arena wall (see Figs. 2-5 for a graphic representation of each stimulus). Both animal and stimulus orders were randomized.

Artificial substrates and objects were made using uniform gray color ( $\mathrm{RGB}=142 ; 50 \%$ gray) designed to elicit a Uniform body pattern, and large black $(\mathrm{RGB}=255)$ and white $(\mathrm{RGB}=0)$ high-contrast checks designed to elicit a Disruptive body pattern (5.5-mm-square size-equal to $100 \%$ of animals' average White Square area). Substrates were computer-generated, laminated to be waterproof, and placed horizontally on the floor under the arena and vertically as a wall inside the arena. 3D objects were $23 \mathrm{~mm}$ in diameter (equal to $1 \mathrm{ML}$ ) and were constructed using the same $50 \%$ gray or black-and-white checks used for the substrate floor and wall.

All 3D objects and 2D wall patterns were positioned in the same place for every animal. A circular 40W fluorescent light source (Phillips CoolWhite) was placed directly above the arena to reduce the effect of shadows. Animals were tested individually and were allowed 10-60 min to acclimate (an animal was considered acclimated when it showed a stable body pattern with little to no motion). After the animal had settled, an image was captured using a remotely controlled camera (Canon Rebel XS).

To examine the visual cues that influence Disruptive body pattern expression, we presented cuttlefish with varying high-contrast elements on 3D freestanding objects (presented on a $50 \%$ gray substrate floor with $50 \%$ gray arena walls) and on 2D vertical walls (presented on a background of $50 \%$ gray wall with a $50 \%$ gray substrate floor). The following characteristics of the substrates were examined: (1) quantity of high-contrast elements on a 3D object and a 2D wall, (2) vertical placement of high-contrast elements on a 3D object and a 2D wall, (3) diameter of a high-contrast 3D object, (4) number of high-contrast 3D objects, and (5) high-contrast substrate on the floor versus wall.

\section{Control experiments}

Two control experiments were performed: (1) uniform $50 \%$ gray substrate floor and arena wall, and (2) checkerboard substrate floor and arena wall (Fig. 1).

\section{Experiment 1: Quantity of high-contrast elements}

Experiment 1a (3D object): Rows of high-contrast checks: 0 (50\% gray object), 2, 4, or 6 checks high were placed on a 3D object (Fig. 2a).

Experiment 1b (2D wall): Similarly, rows of checks: 2,4 , and 6 checks tall ( 4 checks wide) were placed on a 2D wall (Fig. 2b).

\section{Experiment 2: Vertical placement of high-contrast elements}

Experiment 2a (3D object): Two rows of checks were placed at the bottom, middle, and top of a 3D object (Fig. 3a).

Experiment $2 \mathrm{~b}$ (2D wall): Two rows of checks (4 checks 


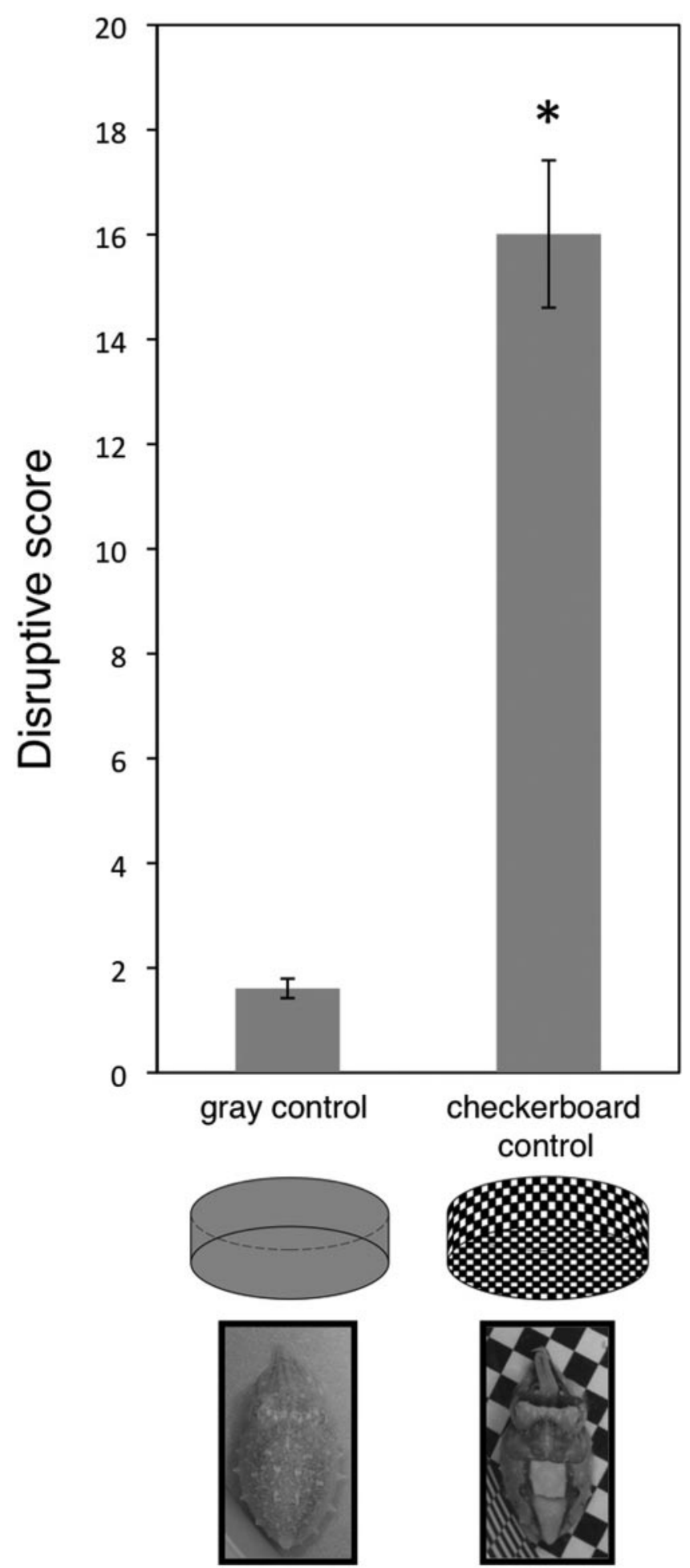

Figure 1. Mean disruptive score of cuttlefish $(n=10)$ on a gray control and a checkerboard control. Bars indicate standard error. Asterisk indicates significant difference from gray control. Cuttlefish images represent the typical body pattern response. wide) were placed at the bottom, middle, and top of a $2 \mathrm{D}$ wall (Fig. 3b).

\section{Experiment 3: Diameter of high contrast 3D object}

3D objects (4 checks tall) with diameters of $1 \mathrm{ML}, 2 \mathrm{ML}$, and $3 \mathrm{ML}$ were presented in the center of the arena (Fig. $4 \mathrm{a}$ ).

\section{Experiment 4: Number of $3 D$ objects}

Differing numbers (1, 2, 3, and 4) of 3D objects (4 checks tall) were presented in the arrangement illustrated in Figure $4 b$.

\section{Experiment 5: High-contrast substrate floor vs. wall}

Cuttlefish were presented with (a) a high-contrast checkerboard substrate floor with a 50\% gray arena wall and (b) a 50\% gray substrate floor with a high-contrast checkerboard wall (Fig. 5).

\section{Image analysis}

We used a MATLAB R2010a (The Mathworks, Inc.) generated image analysis program, developed by C. Chiao, C. Chubb, and L. Siemann, as an automated method for characterizing and discriminating between cuttlefish body patterns. This program runs intensity traces (for more detail see Chiao et al., 2009) along the animal (one medial and three transverse) to estimate activation strengths of 10 Disruptive body pattern components by measuring the difference between the normalized intensity of a component and the normalized intensities of neighboring components. The program generates a "Disruptive Score," which is a summary of the activations of the 10 components.

We used substrates that evoked Uniform or Disruptive body patterns and compared differences in animals' Disruptive Scores using a Kruskal-Wallis ANOVA to analyze body pattern expression. Body patterns evoked in Experiments $1-5$ were compared with body patterns evoked on the gray control. Statistical analyses were performed using MATLAB R2010a.

\section{Results}

\section{Control experiments}

Animals showed a Uniform body pattern on the gray control (i.e., had a low disruptive score; Mean $=1.61, \mathrm{SE}=$ $0.19)$ and a Disruptive body pattern on the checkerboard control (i.e., high disruptive score; Mean $=16.0, \mathrm{SE}=$ 1.41). Cuttlefish body patterns were significantly different on the gray control versus the checkerboard control (Kruskal-Wallis ANOVA, $\chi^{2}=14.29, P=0.002$ ) (Fig. 1). 
a

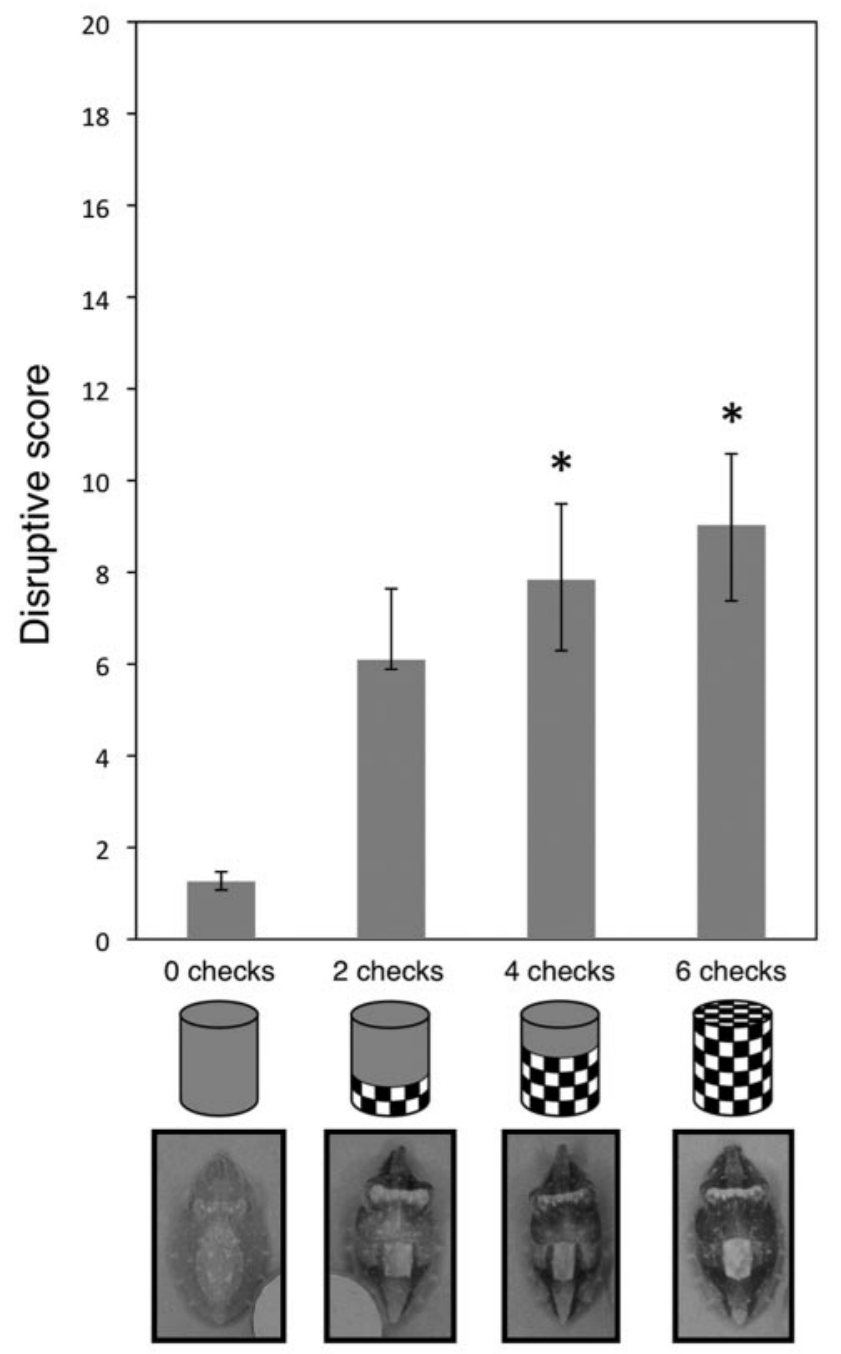

b

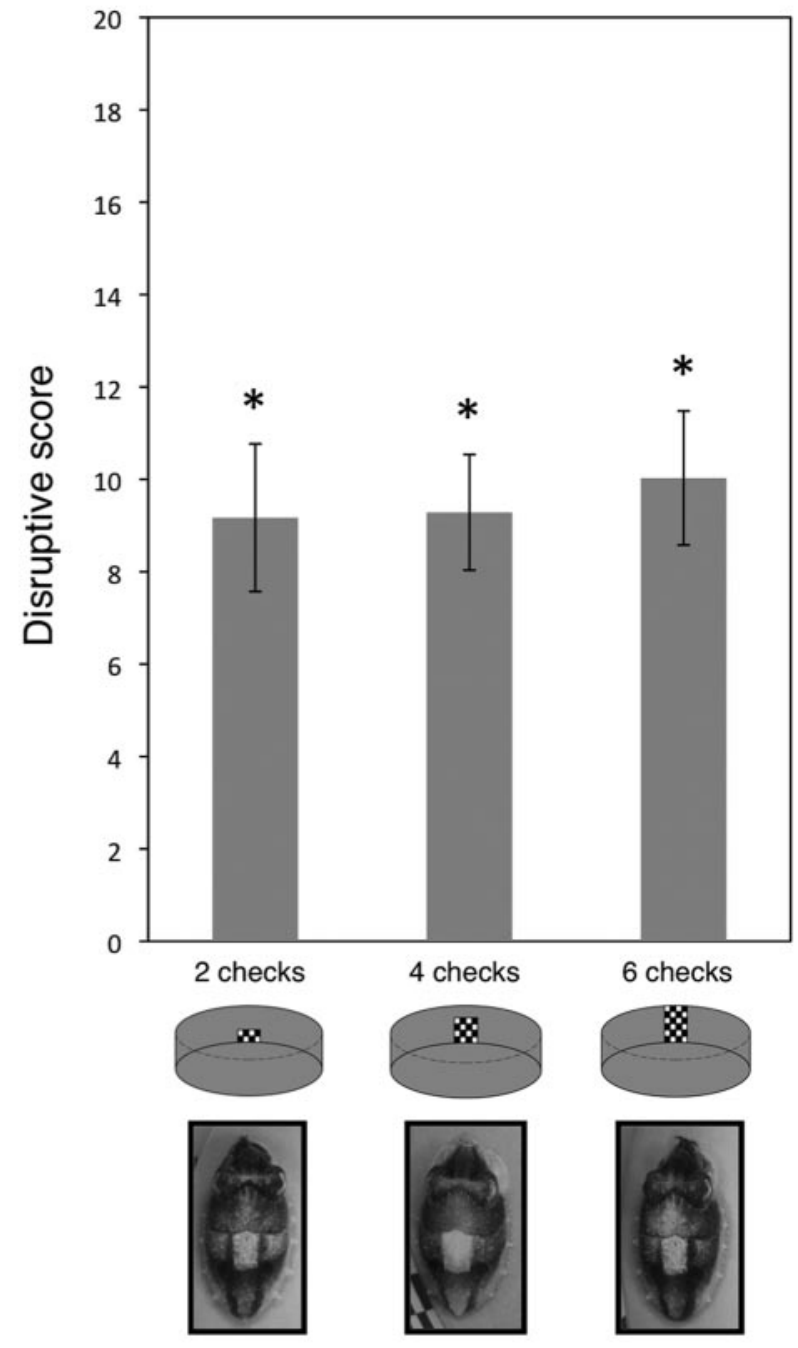

Figure 2. Mean disruptive score of cuttlefish $(n=10)$ for quantity of checks on (a) a 3D object and (b) a 2D wall. Bars indicate standard error. Asterisks indicate significant difference from gray control. Cuttlefish images represent the typical body pattern response.

\section{Experiment 1a: Quantity of high-contrast elements on a} 3 D object (Fig. 2a)

The quantity of high-contrast elements affected cuttlefish body pattern response $\left(\chi^{2}=16.63, P=0.0008\right)$. There was no significant difference between cuttlefish body pattern response to a $50 \%$ gray freestanding $3 \mathrm{D}$ object with no checks (Mean $=1.16, \mathrm{SE}=0.21, P>0.05)$ and the gray control. Cuttlefish responded with a Disruptive body pattern to high-contrast checks on a 3D object $(2,4$, and 6 rows of checks). Pairwise comparisons after a Kruskal-Wallis ANOVA showed no significant difference between cuttlefish body pattern response to 2 rows of checks on a 3D object $($ Mean $=6.10, \mathrm{SE}=1.55, P>0.05)$ and the gray control but showed significant difference between the gray control and 4 rows (Mean $=7.84, \mathrm{SE}=1.65, P<0.05)$ and 6 rows $($ Mean $=9.03, \mathrm{SE}=1.55, P<0.05)$ of checks on a $3 \mathrm{D}$ object.

\section{Experiment 1b: Quantity of high-contrast elements on a $2 D$ wall (Fig. 2b)}

Cuttlefish body pattern responses to a row of 2, 4, or 6 high-contrast checks on a $2 \mathrm{D}$ wall were significantly different from those in the gray control $\left(\chi^{2}=18.28, P=0.0004\right)$. The strength of the Disruptive body pattern expression was not affected by patch size ( 2 rows: Mean $=9.17, \mathrm{SE}=$ 
a

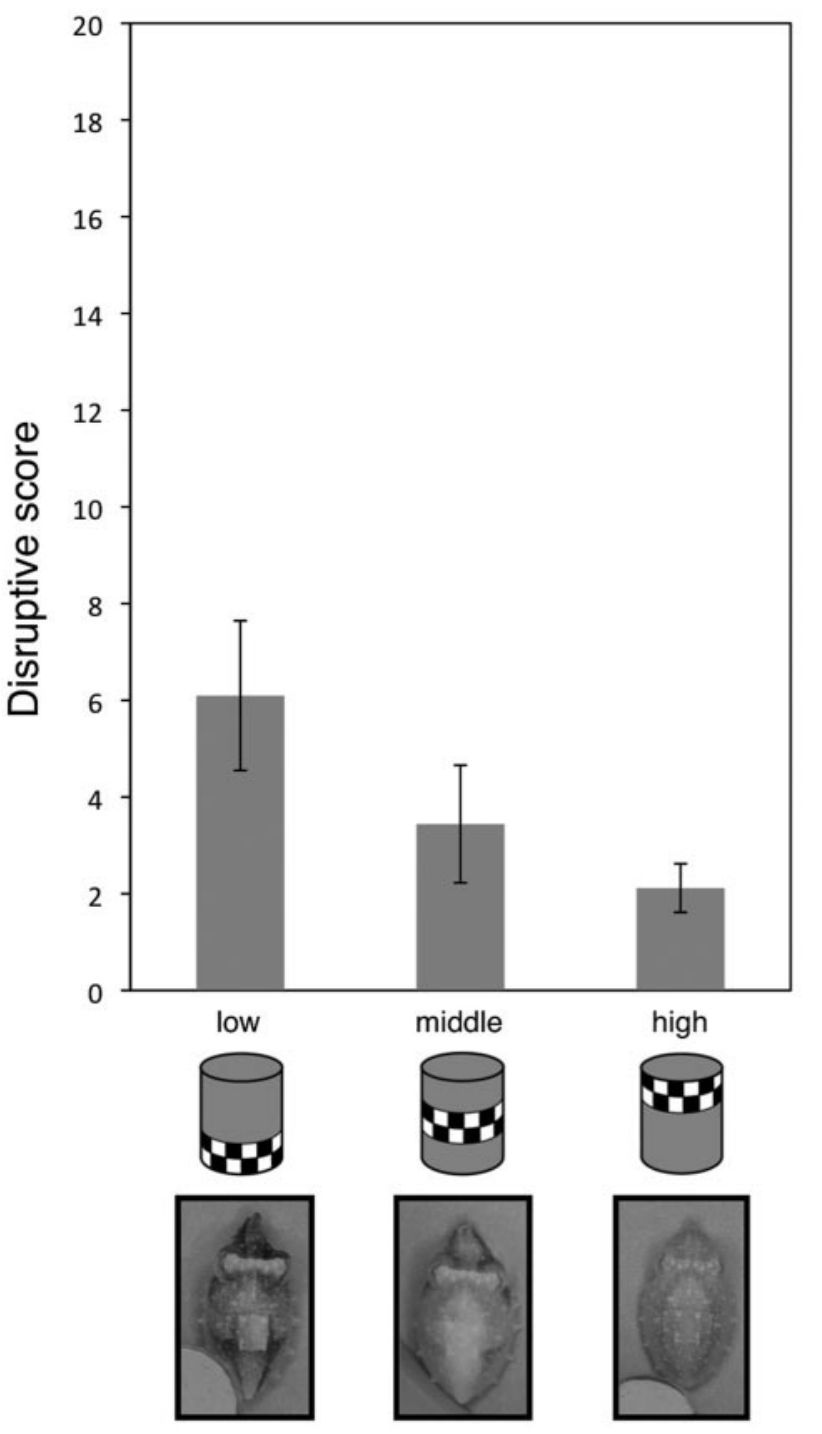

b

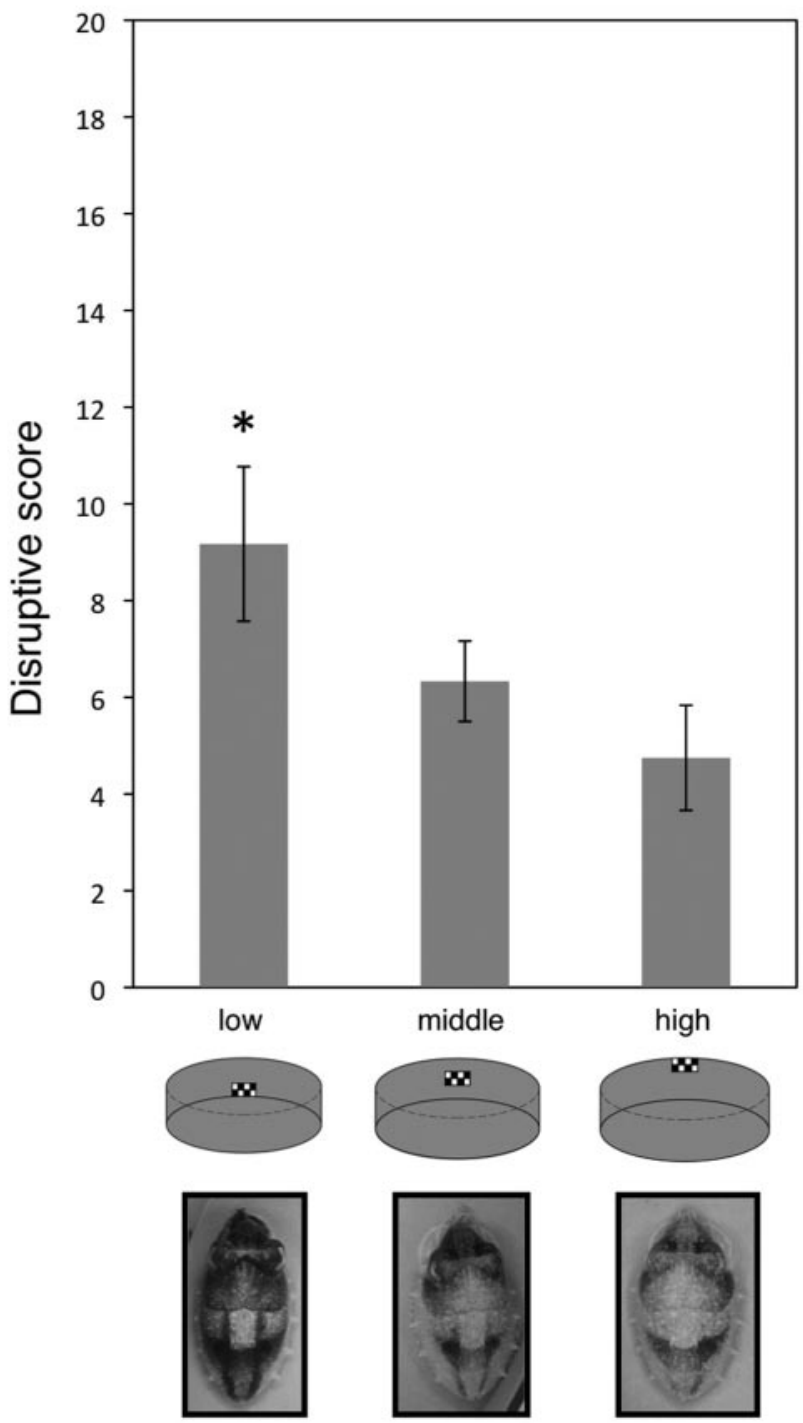

Figure 3. Mean disruptive score of cuttlefish $(n=10)$ for placement (low, middle, high) of two rows of checks on (a) a 3D object and (b) a 2D wall. Bars indicate standard error. Asterisk indicates significant difference from gray control. Cuttlefish images represent the typical body pattern response.

$1.60, P>0.05 ; 4$ rows: Mean $=9.29, \mathrm{SE}=1.25, P>0.05$ 6 rows: Mean $=10.03, \mathrm{SE}=1.45, P>0.05)$.

\section{Experiment 2a: Vertical placement of 2 rows of high-} contrast elements on a 3 D object (Fig. 3a)

Cuttlefish showed a Disruptive body pattern to checks placed low on a 3D object. Animals did not show a Disruptive body pattern when checks were placed in the middle of or high on a 3D object. A Kruskal-Wallis ANOVA showed no significant difference for low, middle, or high placement of checks as compared to the gray control $\left(\chi^{2}=\right.$ 4.51, $P=0.21$ ).
Experiment 2b: Vertical placement of 2 rows of highcontrast elements on a $2 D$ wall (Fig. $3 b$ )

Cuttlefish responded to a patch of two rows of checks when placed in three positions (i.e., low, middle, and high) on a $2 \mathrm{D}$ wall. Disruptive body pattern expression decreased in strength as checks were placed higher on the wall. Cuttlefish body pattern response was significantly different from that in the gray control $\left(\chi^{2}=13.99, P=0.003\right)$ when 2 rows of checks were presented low on the wall (Mean $=$ 9.17, $\mathrm{SE}=1.60$ ), but not when presented in the middle $($ Mean $=6.33, \mathrm{SE}=0.83, P>0.05)$ or high $($ Mean $=4.75$, $\mathrm{SE}=1.09, P>0.05)$ on the wall. 
a

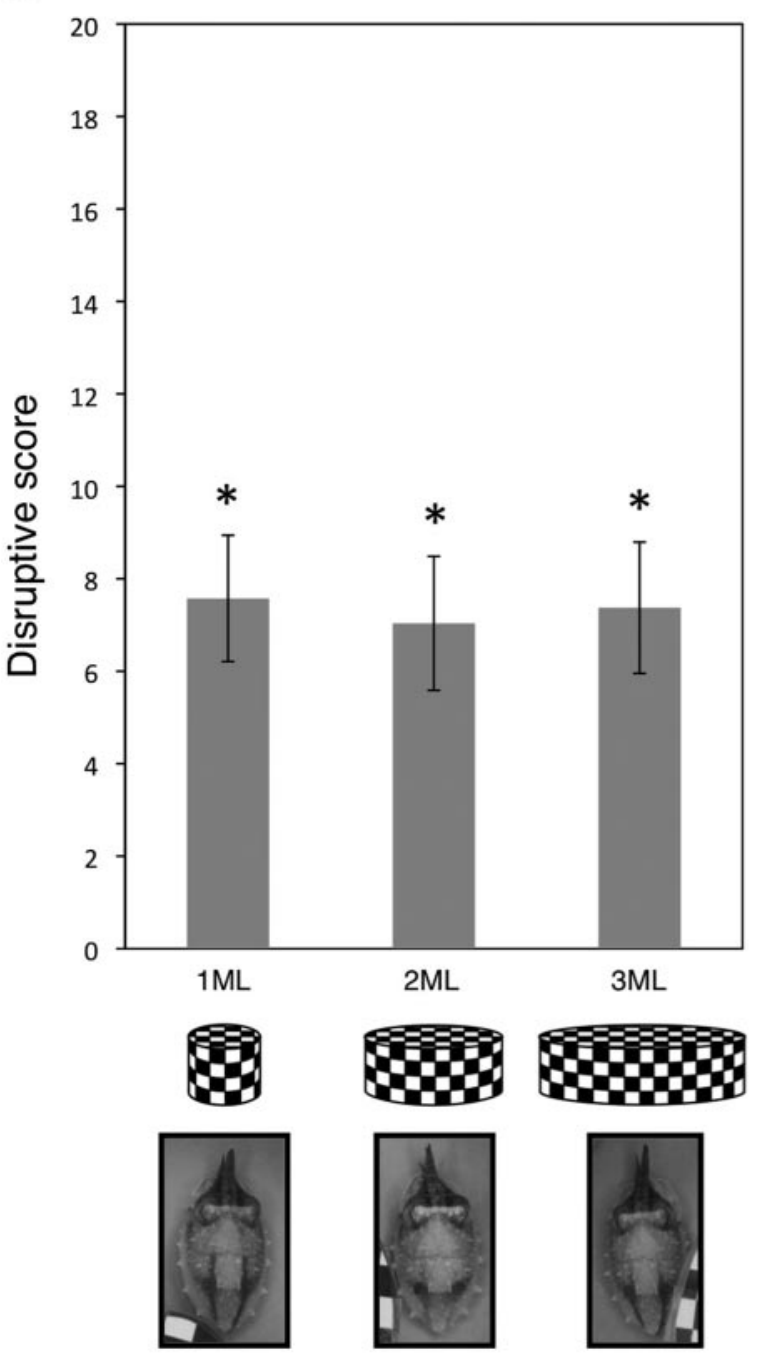

b

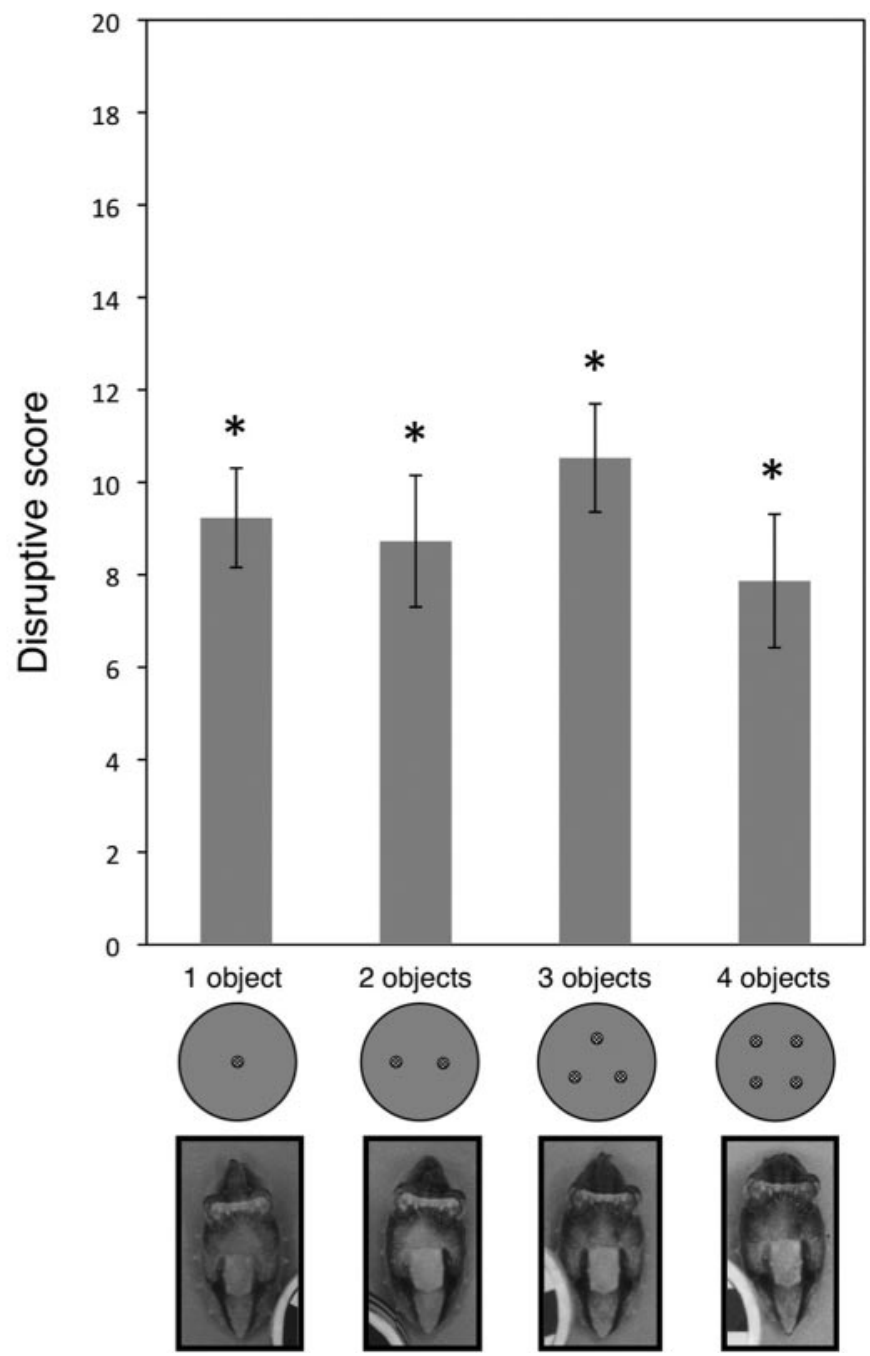

Figure 4. Mean disruptive score of cuttlefish $(n=10)$ for (a) diameter of a 3D object (1 ML-3 ML), (b) numbers of 3D objects (1-4). Bars indicate standard error. Asterisks indicate significant difference from gray control. Cuttlefish images represent the typical body pattern response.

Experiment 3: Diameter of high-contrast 3D object (Fig. $4 a)$

Cuttlefish body pattern response was significantly different from that in the gray control in the presence of a $3 \mathrm{D}$ object (4 checks high) regardless of diameter (1 ML, $2 \mathrm{ML}$, $3 \mathrm{ML})\left(\chi^{2}=14.06, P=0.003\right)$. Increasing the diameter of a high-contrast 3D object did not affect the strength of the animals' Disruptive body pattern expression (1 ML: Mean $=7.58, \mathrm{SE}=1.37, P>0.05 ; 2 \mathrm{ML}$ : Mean $=7.04$, $\mathrm{SE}=1.45, P>0.05 ; 3 \mathrm{ML}:$ Mean $=7.37, \mathrm{SE}=1.42, P$ $>0.05)$.

\section{Experiment 4: Number of 3D objects (Fig. 4b)}

Cuttlefish body pattern response was significantly different from that in the gray control in the presence of 1-4 high-contrast 3D object(s) (all objects were 4 checks high, 1 ML diameter) $\left(\chi^{2}=21.1, P=0.0003\right)$. Increasing the number of high-contrast 3D objects did not affect the strength of the animals' Disruptive body pattern expression ( 1 object: Mean $=9.23, \mathrm{SE}=1.07, P>0.05 ; 2$ objects: Mean $=8.73, \mathrm{SE}=1.42, P>0.05 ; 3$ objects: Mean $=$ $10.53, \mathrm{SE}=1.17, P>0.05 ; 4$ objects: Mean $=7.87, \mathrm{SE}=$ $1.44, P>0.05)$.

\section{Experiment 5: Substrate floor vs. wall (Fig. 5)}

Cuttlefish body pattern response to a checkerboard floor with a gray wall (Mean $=2.51, \mathrm{SE}=0.49, P>0.05$ ) was not significantly different from that in the gray control. Body pattern response to a checkerboard wall with a gray floor $($ Mean $=10.10, \mathrm{SE}=1.31, P<0.05)$ was signifi- 


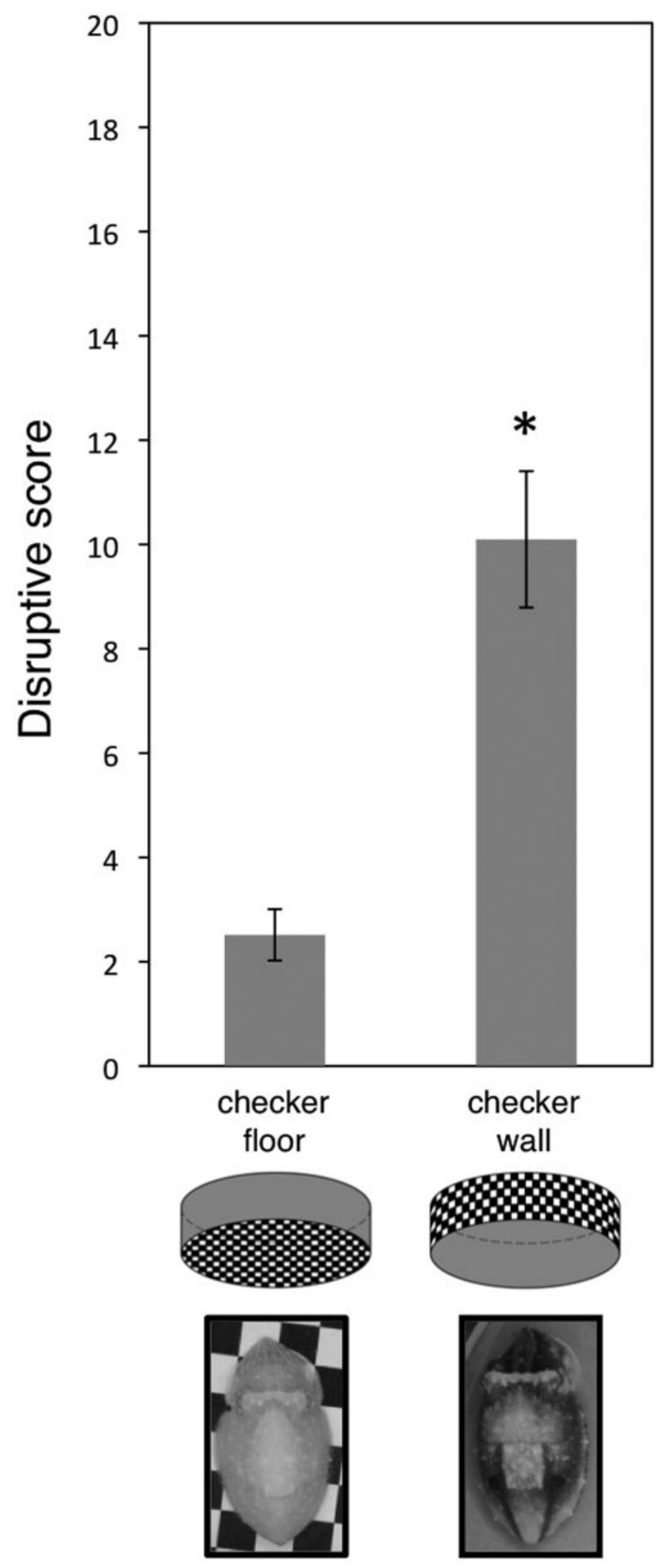

Figure 5. Mean disruptive score of cuttlefish $(n=10)$ on a checkerboard floor with gray wall and on a gray floor with checkered wall. Bars indicate standard error. Asterisk indicates significant difference from gray control. Cuttlefish images represent the typical body pattern response. cantly different from that in the gray control $\left(\chi^{2}=18.4, P\right.$ $=0.0001)$. Our previous laboratory experiments using checkerboard horizontal substrates and black walls evoked disruptive patterns, but as seen in Figure 5, with a gray wall the checkerboard floor evokes only a very minor response of disruptive components.

\section{Discussion}

These laboratory experiments demonstrate that visual stimuli in the vertical dimension (2D or 3D) have a stronger influence on changeable camouflage in Sepia officinalis than do 2D stimuli presented horizontally. Moreover, this effect is noteworthy because in many of the experiments, the stimuli presented vertically constituted only a small proportion of the total visual surrounds, indicating that cuttlefish are selectively responding to vertical cues. Thus, vertical orientation should be added to the list of key visual features known to drive cuttlefish camouflage patterning (i.e., size, contrast, and edges: Chiao and Hanlon, 2001a; Chiao et al., 2005; Barbosa et al., 2008b; Zylinski et al., 2009b, c, 2011).

Cuttlefish responded with a significantly different body pattern to high-contrast $3 \mathrm{D}$ objects if there were at least 4 rows of checks on the object (Fig. 2a). Increasing the diameter or number of the 3D object did not increase the strength of the response (Fig. 4a, b), indicating that a stimulus as small as 16 black-and-white checks is enough to evoke a Disruptive body pattern. Interestingly, cuttlefish always responded to high-contrast checks on a 2D wall, regardless of the quantity or placement of the stimulus (Fig $2 \mathrm{~b}, 3 \mathrm{~b}$ ). Taken together, these laboratory experiments demonstrate the strong influence of vertically oriented visual stimuli.

A previous study showed that cuttlefish respond to both vertical and horizontal cues for camouflage, but did not address the relative influence of horizontal versus vertical orientation (Barbosa et al., 2008a). Our results suggest that vertical cues take precedence over horizontal cues when cuttlefish are presented with high-contrast stimuli because cuttlefish responded more strongly to a checkerboard covering the entire wall than to a checkerboard covering the entire substrate floor (Fig. 5). Similarly, Buresch et al. (2011) found that cuttlefish did not respond to a 2D highcontrast patch (16 black-and-white checks on 50\% gray) presented horizontally on the substrate floor. Additionally, in a separate experiment using vertically oriented cues, cuttlefish responded by raising their arms relative to the angle of a vertical stimulus (Barbosa et al., 2012).

Since it appears as though cuttlefish are responding primarily to vertical cues, irrespective of whether they are presented on a discrete object, can we interpret our results as "masquerade camouflage"? It is not possible to definitively prove that this type of camouflage is masquerade, since this would require viewing the animals from the perspective of 
a predator rather than human vision (Stevens and Merilaita, 2009; Skelhorn et al., 2010a). However, cuttlefish in the wild use body posture and skin papillae in addition to chromatic body pattern to resemble inanimate objects in their surroundings (Hanlon et al., 2009). This type of behavior fits into the recent definition of masquerade: looking like an inedible or inanimate object (Skelhorn et al., 2010a, 2011; Skelhorn and Ruxton, 2011). It is not surprising that cuttlefish camouflage to vertical structures in their environment because predators view these animals from many different angles: pelagic predators from above and benthic predators from the side.

Although masquerade is the most plausible explanation for the behavior seen in these experiments, cuttlefish could be responding with body patterns that match the spaceaveraged contrast and spatial frequencies in their visual surroundings. The Disruptive patterns in the presence of checkerboard cues on a uniform background were weaker than those of cuttlefish on the checkerboard control. In laboratory experiments with split (left side $v s$. right side) substrates, cuttlefish integrated conflicting visual cues and responded with mixed body patterns (Allen et al., 2010). While these split-substrate experiments indicate that cuttlefish may average visual cues when choosing a pattern, our data seem to indicate that cuttlefish are selectively responding to a small percentage of their visual field. Other cephalopods have been shown to selectively sample visual features for body patterning. Two octopus species (Octopus cyanea and Octopus vulgaris) base some of their body patterns on features of nearby objects rather than on their entire field of view (Josef et al., 2012). It is also possible that both masquerade and background matching are occurring simultaneously and working together to enhance camouflage, as previously demonstrated in caterpillars and lizards (Skelhorn et al., 2011; Cooper and Sherbrooke, 2012).

Additional field work and laboratory experiments involving 3D objects could help us better understand masquerade as a camouflage tactic used by cuttlefish. For example, detailed field measurements of cuttlefish reflectance compared to that of adjacent objects may yield precise details of the visual sampling rules that guide cuttlefish camouflage in diverse habitats; efforts in this direction have recently been made with point-by-point spectrophotometric measurements and hyper-spectral imaging (Chiao et al., 2011; Akkaynak et al., 2012). Although the scope of this study was restricted to experimental conditions in the laboratory, we have demonstrated that vertical cues strongly influence cuttlefish body pattern choice.

\section{Acknowledgments}

We thank the animal care staff in the Marine Resources Center for help with weekend care of our cuttlefish colony. Charlie Chubb was helpful with statistical analysis. We thank Sarah Zylinski and an anonymous reviewer for their helpful reviews of this manuscript, and the editor for providing valuable comments. This work was funded by the United States Department of Defense (grant number W911NF-07-D-0001).

\section{Literature Cited}

Akkaynak, D., J. J. Allen, L. M. Mäthger, C.-C. Chiao, and R. T. Hanlon. 2012. Quantification of cuttlefish (Sepia officinalis) camouflage: a study of color and luminance using in situ spectrometry. J. Comp. Physiol. A 199: 1-15.

Allen, J. J., L. M. Mäthger, A. Barbosa, and R. T. Hanlon. 2009. Cuttlefish use visual cues to control 3-dimensional skin papillae for camouflage. J. Comp. Physiol. A 195: 547-555.

Allen, J. J., L. M. Mäthger, A. Barbosa, K. C. Buresch, E. Sogin, J. Schwartz, C. Chubb, and R. T. Hanlon. 2010. Cuttlefish dynamic camouflage: responses to substrate choice and integration of multiple visual cues. Proc. R. Soc. B 277: 1031-1039.

Barbosa, A., L. M. Mäthger, C. Chubb, C. Florio, C. C. Chiao, and R. T. Hanlon. 2007. Disruptive coloration in cuttlefish: a visual perception mechanism that regulates ontogenetic adjustment of skin patterning. J. Exp. Biol. 210: 1139-1147.

Barbosa, A., L. Litman, and R. T. Hanlon. 2008a. Changeable cuttlefish camouflage is influenced by horizontal and vertical aspects of the visual background. J. Comp. Physiol. A 194: 405-413.

Barbosa, A., L. M. Mäthger, K. C. Buresch, J. Kelly, C. Chubb, C.-C. Chiao, and R. T. Hanlon. 2008b. Cuttlefish camouflage: the effects of substrate contrast and size in evoking uniform, mottle or disruptive body patterns. Vision Res. 48: 1242-1253.

Barbosa, A., J. J. Allen, L. M. Mäthger, and R. T. Hanlon. 2012. Cuttlefish use visual cues to determine arm postures for camouflage Proc. R. Soc. B 279: 84-90.

Boletzky, S. v. 1983. Sepia officinalis. Pp. 31-52 in Cephalopod Life Cycles, vol. 1, P. R. Boyle, ed. Academic Press, London.

Buresch, K. C., L. M. Mäthger, J. J. Allen, C. Bennice, N. Smith, J. Schram, C.-C. Chiao, C. Chubb, and R. T. Hanlon. 2011. The use of background matching versus masquerade for camouflage in cuttlefish Sepia officinalis. Vision Res. 51: 2362-2368.

Chiao, C.-C., and R. T. Hanlon. 2001a. Cuttlefish camouflage: visual perception of size, contrast and number of white squares on artificial checkerboard substrata initiates disruptive coloration. J. Exp. Biol. 204: 2119-2125.

Chiao, C.-C., and R. T. Hanlon. 2001b. Cuttlefish cue visually on area-not shape or aspect ratio- of light objects in the substrate to produce disruptive body patterns for camouflage. Biol. Bull. 201: 269-270.

Chiao, C.-C., E. J. Kelman, and R. T. Hanlon. 2005. Disruptive body patterning of cuttlefish (Sepia officinalis) requires visual information regarding edges and contrast of objects in natural substrate backgrounds. Biol. Bull. 208: 7-11.

Chiao, C.-C., C. Chubb, and R. T. Hanlon. 2007. Interactive effects of size, contrast, intensity and configuration of background objects in evoking disruptive camouflage in cuttlefish. Vision Res. 47: 22232235

Chiao, C.-C., C. Chubb, K. Buresch, and L. Siemann. 2009. The scaling effects of substrate texture on camouflage patterning in cuttlefish. Vision Res. 49: 1647-1656.

Chiao, C.-C., J. K. Wickiser, J. J. Allen, B. Genter, and R. T. Hanlon. 2011. Hyperspectral imaging of cuttlefish camouflage indicates good color match in the eyes of fish predators. Proc. Natl. Acad. Sci. USA 108: $9148-9153$.

Cooper, W. E., Jr., and W. C. Sherbrooke. 2012. Choosing between 
a rock and a hard place: camouflage in the round-tailed horned lizard Phrynosoma modestum. Curr. Zool. 58: 541-548.

Cott, H. B. 1940. Adaptive Coloration in Animals. Methuen, London.

Endler, J. A. 1978. A predator's view of animal color patterns. Evol. Biol. 11: 319-364.

Endler, J. A. 1981. An overview of the relationships between mimicry and crypsis. Biol. J. Linn. Soc. 16: 25-31.

Hanlon, R. T., and J. B. Messenger. 1988. Adaptive coloration in young cuttlefish (Sepia officinalis L.): the morphology and development of body patterns and their relation to behaviour. Philos. Trans. $R$. Soc. Lond. B 320: 437-487.

Hanlon, R. T., and J. B. Messenger. 1996. Cephalopod Behaviour. Cambridge University Press, Cambridge.

Hanlon, R. T., C.-C. Chiao, L. M. Mäthger, A. Barbosa, K. C. Buresch, and C. Chubb. 2009. Cephalopod dynamic camouflage: bridging the continuum between background matching and disruptive coloration. Philos. Trans. R. Soc. Lond. B 364: 429-437.

Josef, N., P. Amodio, G. Fiorito, and N. Shashar. 2012. Camouflaging in a complex environment-octopuses use specific features of their surroundings for background matching. PLoS ONE 7: e37579.

Kelman, E. J., R. J. Baddeley, A. J. Shohet, and D. Osorio. 2007. Perception of visual texture and the expression of disruptive camouflage by the cuttlefish, Sepia officinalis. Proc. R. Soc. B 274: 13691375.

Mäthger, L. M., A. Barbosa, S. Miner, and R. T. Hanlon. 2006. Color blindness and contrast perception in cuttlefish (Sepia officinalis) determined by a visual sensorimotor assay. Vision Res. 46: 1746-1753.

Mäthger, L. M., C. C. Chiao, A. Barbosa, K. C. Buresch, S. Kaye, and R. T. Hanlon. 2007. Disruptive coloration elicited on controlled natural substrates in cuttlefish, Sepia officinalis. J. Exp. Biol. 210: 2657-2666.

Ruxton, G. D., T. N. Sherratt, and M. P. Speed. 2005. Avoiding Attack: The Evolutionary Ecology of Crypsis, Warning Signals and Mimicry. Oxford University Press, Oxford.
Schmidt, J. O. 1990. Insect Defenses: Adaptive Mechanisms and Strategies of Prey and Predators. SUNY Press, Albany, NY.

Shohet, A. J., R. J. Baddeley, J. C. Anderson, E. J. Kelman, and D. Osorio. 2006. Cuttlefish responses to visual orientation of substrates, water flow and a model of motion camouflage. J. Exp. Biol. 209: 4717-4723.

Skelhorn, J., and G. D. Ruxton. 2011. Mimicking multiple models: polyphenetic masqueraders gain additional benefits from crypsis. Behav. Ecol. 22: 60-65.

Skelhorn, J., H. M. Rowland, and G. D. Ruxton. 2010a. The evolution and ecology of masquerade. Biol. J. Linn. Soc. 99: 1-8.

Skelhorn, J., H. M. Rowland, M. P. Speed, and G. D. Ruxton. 2010b. Masquerade: camouflage without crypsis. Science 327: 51.

Skelhorn, J., H. M. Rowland, J. Delf, M. P. Speed, and G. D. Ruxton. 2011. Density-dependent predation influences the evolution and behavior of masquerading prey. Proc. Natl. Acad. Sci. USA 108: 65326536

Stevens, M., and S. Merilaita. 2009. Animal camouflage: current issues and new perspectives. Philos. Trans. R. Soc. Lond. B 364: 423-427.

Stevens, M., and S. Merilaita. 2011. Animal Camouflage: Mechanisms and Function. Cambridge University Press, Cambridge.

Thayer, G. H., and A. H. Thayer. 1918. Concealing-Coloration in the Animal Kingdom. MacMillan, New York.

Zylinski, S., D. Osorio, and A. J. Shohet. 2009a. Cuttlefish camouflage: context-dependent body pattern use during motion. Proc. R. Soc B 276: 3963-3969.

Zylinski, S., D. Osorio, and A. J. Shohet. 2009b. Edge detection and texture classification by cuttlefish. J. Vis. 9: 1-10.

Zylinski, S., D. Osorio, and A. J. Shohet. 2009c. Perception of edges and visual texture in the camouflage of the common cuttlefish, Sepia officinalis Philos. Trans. R. Soc. Lond. B 364: 439-448.

Zylinski, S., M. J. How, D. Osorio, R. T. Hanlon, and N. J. Marshall. 2011. To be seen or to hide: visual characteristics of body patterns for camouflage and communication in the Australian giant cuttlefish Sepia apama. Am. Nat. 177: 681-690. 